\title{
Euler Matrix Method for Solving Complex Differential Equations with Variable Coefficients in Rectangular Domains
}

\author{
Necdet Bildik*, Mehtap Tosun, Sinan Deniz \\ Celal Bayar University, Faculty of Arts \& Science, Department of Mathematics, Muradiye Campus, 45030, \\ Manisa, Turkey. \\ * Corresponding author. Tel.: 00900236201 3203; email: necdet.bildik@cbu.edu.tr \\ Manuscript submitted April 25, 2016; accepted September 30, 2016. \\ doi: 10.17706/ijapm.2017.7.1.69-78
}

\begin{abstract}
In this study, we examine the approximate solutions of complex differential equations in rectangular domains by using Euler polynomials. We construct the matrix forms of Euler polynomials and their derivatives to transform the considered differential equation to matrix equation with unknown Euler coefficients. This matrix equation is also equivalent to a system of linear algebraic equations. Linear system is solved by substituting collocation points into those matrix forms to get the unknown Euler coefficients. Determining these coefficients provides the approximate solutions of the given complex differential equations under the given conditions.
\end{abstract}

Key words: Euler polynomials, complex differential equations, collocation method.

\section{Introduction}

Do not change the font sizes or line spacing to squeeze more text into a limited number of pages. Use italics for emphasis; do not underline.

Complex differential equations arise from many important applications in physics, engineering, applied science, etc. Vibrations of a one-mass system with two DOFs (degree of freedom) are a good example to illustrate the one of the many implementations of them. Complex differential equations have been tried to solve by some techniques [1]-[4]. However, it is not always possible to have the solution of these differential equations explicitly. So, researchers need some numerical techniques to cope with difficulties generated from the structure of complex differential equations. Collocation methods are one of the well-known methods for solving many differential equations [5]-[10] and they are also useful for complex differential equations.

This paper offers Euler matrix method for solving linear complex differential equations with variable coefficients in a rectangular domain such as:

$$
\begin{aligned}
& f^{(m)}(z)+\sum_{k=0}^{m-1} P_{k}(z) f^{(k)}(z)=S(z) \\
& m \geq 1, z=x+i y, x \in[a, b], y \in[c, d]
\end{aligned}
$$

under the conditions: 


$$
f^{(r)}(0)=\alpha_{r}, r=0,1, \ldots, m-1
$$

Here we assume that the coefficients $P_{k}(z)$, known function $S(z)$ and unknown function $f(z)$ are analytic functions in the rectangular domain $D=\{z \in C: z=x+i y, a \leq x \leq b, c \leq y \leq d: a, b, c, d \in R\}$ where the coefficients $\alpha_{r}$ are suitable constants. We assume that the solution of Eq. (1) under the given conditions (2) is given in the form:

$$
f(z) \approx f_{N}(z)=\sum_{n=0}^{N} f_{n} E_{n}(z)
$$

which is the truncated Euler series of the unknown function $f(z)$. In order to determine the Euler coefficients $f_{n}$, we use the collocation points [5], [6]:

$$
z_{p p}=x_{p}+i y_{p}
$$

where

$$
x_{p}=a+\frac{b-a}{N} p, y_{p}=c+\frac{d-c}{N} p, p=0,1, \ldots, N .
$$

\section{Revision of Euler Polynomials and Operational Matrix Review Stage}

The classical Euler polynomials $E_{n}(x)$ are defined as [11]-[13]:

$$
e^{E(x) t}=\sum_{n=0}^{\infty} E_{n}(x) \frac{t^{n}}{n !}=\frac{2}{e^{t}+1} e^{x t},|t|<\pi
$$

The first few Euler polynomials are:

$$
\begin{aligned}
& E_{0}(x)=1 \\
& E_{1}(x)=x-\frac{1}{2} \\
& E_{2}(x)=x^{2}-x \\
& E_{3}(x)=x^{3}-\frac{3}{2} x^{2}+\frac{1}{4} \\
& E_{4}(x)=x^{4}-2 x^{3}+x \\
& E_{5}(x)=x^{5}-\frac{5}{2} x^{4}+\frac{5}{2} x^{2}-\frac{1}{2} \\
& E_{6}(x)=x^{6}-3 x^{5}+5 x^{3}-3 x
\end{aligned}
$$

Some basic properties about these polynomials are as follows: 


$$
\begin{aligned}
& \sum_{k=0}^{n}\left(\begin{array}{l}
n \\
k
\end{array}\right) E_{k}(x)+E_{n}(x)=2 x^{n} \\
& E_{n}(x+y)=\sum_{k=0}^{n}\left(\begin{array}{l}
n \\
k
\end{array}\right) E_{k}(x) y^{n-k} \\
& E_{N}(x)=\sum_{n=0}^{N}\left(\begin{array}{l}
N \\
n
\end{array}\right) E_{n}(0) x^{N-n}
\end{aligned}
$$

They also satisfy the relations:

$$
\begin{gathered}
\frac{d E_{n}() x}{d x}=n E_{n-1}() x,(\geq n) 1 \\
\int_{0}^{1} E_{n}(x) d x=-\frac{2 E_{n+1}}{n+1} \\
E_{n}(x+1)+E_{n}(x)=2 x^{n} \\
\sum_{k=0}^{n}\left(\begin{array}{l}
n \\
k
\end{array}\right) E_{k}(x) E_{n-k}(w)=2(1-w-z) E_{n}(z+w)+2 E_{n+1}(z+w), n \geq 0
\end{gathered}
$$

and also the following differential equation:

$$
\frac{E_{n}(0)}{n !} y^{(n)}(x)+\frac{E_{n-1}(0)}{(n-1) !} y^{(n-1)}(x)+\cdots+\frac{E_{2}(0)}{2 !} y^{\prime \prime}(x)+\left(\frac{1}{2}-x\right) y^{\prime}(x)+n y(x)=0
$$

If we define the Euler vector $E(x)$ in the form $E(x)=\left[E_{0}(x), E_{1}(x), E_{2}(x), \cdots, E_{n}(x)\right]$, we can write the following relation by means of the property (9)

$$
\underbrace{\left[\begin{array}{c}
E_{0}^{\prime}(x) \\
E_{1}^{\prime}(x) \\
E_{2}^{\prime}(x) \\
\vdots \\
E_{n-1}^{\prime}(x) \\
E_{n}^{\prime}(x)
\end{array}\right]}_{E^{\prime}(x)^{T}}=\underbrace{\left[\begin{array}{lllllll}
0 & 0 & 0 & \cdots & 0 & 0 & 0 \\
1 & 0 & 0 & \cdots & 0 & 0 & 0 \\
0 & 2 & 0 & \cdots & 0 & 0 & 0 \\
\vdots & \vdots & \vdots & \ddots & \vdots & \vdots & \vdots \\
0 & 0 & 0 & \cdots & N-1 & 0 & 0 \\
0 & 0 & 0 & \cdots & 0 & N & 0
\end{array}\right]}_{M} \underbrace{\left[\begin{array}{l}
E_{0}(x) \\
E_{1}(x) \\
E_{2}(x) \\
\vdots \\
E_{n-1}(x) \\
E_{n}(x)
\end{array}\right] .}_{E^{T}}
$$

$M$ is called the operational matrix of differentiation. Note that if we use the complex variable $z$ instead of the real variable $x$ in the matrix relation (13), we get the same result since the well-known property $\left(z^{n}\right)^{\prime}=n z^{n-1}$. Hence, $k$ th derivative of $E(x)$ can be constructed as:

$$
\begin{aligned}
& E^{\prime}(x)^{T}=M E(x)^{T} \\
& \Rightarrow E^{(1)}(x)=E(x) M^{T} \\
& E^{(2)}(x)=E^{(1)}(x) M^{T}=E(x)\left(M^{T}\right)^{2} \\
& E^{(3)}(x)=E^{(1)}(x)\left(M^{T}\right)^{2}=E(x)\left(M^{T}\right)^{3} \\
& E^{(k)}(x)=E(x)\left(M^{T}\right)^{k} .
\end{aligned}
$$




\section{Method of Solution}

Let us consider the complex differential equation with variable coefficients (1) and the truncated series or its approximated solution $f_{N}(z)$. Evidently, $f_{N}(z)$ can be written as:

$$
f_{N}(z)=E(z) F
$$

where

$$
\begin{aligned}
& E(z)=\left[\begin{array}{llll}
E_{0}(z) & E_{1}(z) & \cdots & E_{N}(z)
\end{array}\right] \\
& F=\left[\begin{array}{llll}
f_{0} & f_{1} & \cdots & f_{N}
\end{array}\right]^{T}
\end{aligned}
$$

Thus, by considering the Eq.(14) we can get

$$
f_{N}^{(k)}(z)=E(z)\left(M^{T}\right)^{k} F, \quad \mathrm{k} \leq N
$$

Using the collocation points $z=z_{p p}(p=0,1, \cdots, N)$, the matrix relation (17) becomes

$$
\begin{aligned}
f_{N}^{(k)}\left(z_{00}\right) & =E\left(z_{00}\right)\left(M^{T}\right)^{k} F, \\
f_{N}^{(k)}\left(z_{11}\right) & =E\left(z_{11}\right)\left(M^{T}\right)^{k} F, \\
\vdots & \\
f_{N}^{(k)}\left(z_{N N}\right) & =E\left(z_{N N}\right)\left(M^{T}\right)^{k} F .
\end{aligned}
$$

where

$$
E\left(z_{p p}\right)=\left[\begin{array}{llll}
E_{0}\left(z_{p p}\right) & E_{1}\left(z_{p p}\right) & \cdots & E_{N}\left(z_{p p}\right)
\end{array}\right]
$$

or briefly

$$
F^{(k)}=\left[\begin{array}{c}
f_{N}^{(k)}\left(z_{00}\right) \\
f_{N}^{(k)}\left(z_{11}\right) \\
\vdots \\
f_{N}^{(k)}\left(z_{N N}\right)
\end{array}\right]=L\left(M^{T}\right)^{k} F
$$

where

$$
\begin{aligned}
L & =\left[\begin{array}{c}
E\left(z_{00}\right) \\
E\left(z_{11}\right) \\
\vdots \\
E\left(z_{N N}\right)
\end{array}\right] \\
& =\left[\begin{array}{lllll}
E_{0}\left(z_{00}\right) & E_{1}\left(z_{00}\right) & E_{2}\left(z_{00}\right) & \ldots & E_{N}\left(z_{00}\right) \\
E_{0}\left(z_{11}\right) & E_{1}\left(z_{11}\right) & E_{2}\left(z_{11}\right) & \ldots & E_{N}\left(z_{11}\right) \\
\vdots & \vdots & \vdots & \ddots & \vdots \\
E_{0}\left(z_{N N}\right) & E_{1}\left(z_{N N}\right) & E_{2}\left(z_{N N}\right) & \ldots & E_{N}\left(z_{N N}\right)
\end{array}\right]
\end{aligned}
$$


Substituting the collocation points $z=z_{p p}$ into Eq. (1) yields

$$
f_{N}^{(m)}\left(z_{p p}\right)+\sum_{k=0}^{m-1} P_{k}\left(z_{p p}\right) f_{N}^{(k)}\left(z_{p p}\right)=S\left(z_{p p}\right), \quad p=0,1, \cdots, N
$$

or

$$
F^{(m)}+\sum_{k=0}^{m-1} P_{k} F^{(k)}=L\left(M^{T}\right)^{m}+\sum_{k=0}^{m-1} P_{k} L\left(M^{T}\right)^{k} F=S
$$

where

$$
S=\left[\begin{array}{llll}
S\left(z_{00}\right) & S\left(z_{11}\right) & \cdots & S\left(z_{N N}\right)
\end{array}\right]^{T}
$$

And

$$
P_{k}=\left[\begin{array}{cccc}
P_{k}\left(z_{00}\right) & 0 & \cdots & 0 \\
0 & P_{k}\left(z_{11}\right) & \cdots & 0 \\
\vdots & \vdots & \ddots & \vdots \\
0 & 0 & \cdots & P_{k}\left(z_{N N}\right)
\end{array}\right]
$$

We now consider fundamental matrix Eq. (23) corresponding to Eq. (1). Eq. (23) can be rewritten in the form as:

$$
W F=S \quad \text { or }[W ; S]=\left[w_{p q} ; s_{p}\right] \quad p, q=0,1, \cdots, N
$$

where

$$
W=L\left(M^{T}\right)^{m}+\sum_{k=0}^{m-1} P_{k} L\left(M^{T}\right)^{k}
$$

One can also obtain the corresponding matrix form for the given conditions (2) by using the relation (17) as follows:

$$
f^{(r)}(0)=E(0)\left(M^{T}\right)^{r} F=\alpha_{r}, \quad r=0,1, \cdots, m-1
$$

or in vector form

$$
U_{r} F=\alpha_{r}
$$

where

$$
U_{r}=E(0)\left(M^{T}\right)^{r}, r=0,1, \cdots, m-1 .
$$


Equivalently, it can be written as the augmented matrix form:

$$
\left[U_{r} ; \alpha_{r}\right]=\left[\begin{array}{llllll}
u_{r 0} & u_{r 1} & u_{r 2} & \ldots & u_{r N} & ;
\end{array} \alpha_{r}\right], \quad r=0,1, \cdots, m-1 .
$$

Eventually, we replace the $m$ row matrices (31) by the last $m$ rows of the augmented matrix (27) to get the unknown Euler coefficients $f_{n}, n=0,1, \cdots, N$ to obtain the approximate solution of the problem consisting of Eq. (1) and the conditions (2). By doing so, we have new augmented matrix

$$
[\hat{W} ; \hat{S}]=\left[\begin{array}{llllll}
w_{00} & w_{01} & \ldots & w_{0 N} & ; & s_{0} \\
w_{10} & w_{11} & \ldots & w_{1 N} & ; & s_{1} \\
\vdots & \vdots & \ddots & \vdots & \vdots & \vdots \\
w_{N-m, 0} & w_{N-m, 1} & \ldots & w_{N-m, N} & ; & s_{N-m} \\
u_{00} & u_{01} & \ldots & u_{0 N} & ; & \alpha_{0} \\
u_{10} & u_{11} & \ldots & u_{1 N} & ; & \alpha_{1} \\
\vdots & \vdots & \ldots & \vdots & \vdots & \vdots \\
u_{m-1,0} & u_{m-1,1} & \ldots & u_{m-1, N} & ; & \alpha_{m-1}
\end{array}\right]
$$

or the corresponding matrix-vector equation

$$
\hat{W} F=\hat{S}
$$

If $\operatorname{det}(\hat{W}) \neq 0$, we can write (32) as

$$
F=(\hat{W})^{-1} \hat{S}
$$

and the matrix $F$ can be uniquely determined. Thus the $m t h$-order complex differential equation with variable coefficients (1) with the given conditions (2) has a unique solution. This solution is given by the truncated Euler series (3). For stability analysis of differential equations, we refer to [14]

\section{Examples}

Example 1. Consider the linear first order complex differential equation (5)

$$
f^{\prime}(z)+z f(z)=2 z^{2}-z+2, z=x+i y, x \in[0,1], \mathrm{y} \in[0,1]
$$

with $f(0)=-1$.

Collocation points for $N=3$ are

$$
z_{00}=0, \mathrm{z}_{11}=(1+i) / 3, \mathrm{z}_{22}=(2+2 i) / 3 \text { and } \mathrm{z}_{33}=1+\mathrm{i}
$$

We need to find the Euler coefficients $f_{0}, f_{1}, f_{2}, f_{3}$ by accepting the approximate solution as in the form 
of Eq. 3

$$
f_{3}(z)=\sum_{n=0}^{3} f_{n} E_{n}(z), z \in \mathrm{D}
$$

We have $P_{0}(z)=z$ and $S(z)=2 z^{2}-z+2$, then

$$
\begin{gathered}
P_{0}=\left[\begin{array}{cccc}
0 & 0 & 0 & 0 \\
0 & 0.3333+0.3333 i & 0 & 0 \\
0 & 0 & 0.6667+0.6667 i & 0 \\
0 & 0 & 0 & 1.0000
\end{array}\right], \\
S=\left[\begin{array}{l}
S\left(z_{00}\right) \\
S\left(z_{11}\right) \\
S\left(z_{22}\right) \\
S\left(z_{33}\right)
\end{array}\right]=\left[\begin{array}{c}
2.0000 \\
1.6667+0.1111 i \\
1.3333+1.1111 i \\
1.0000+3.0000 i
\end{array}\right]
\end{gathered}
$$

and for $N=3$ we get

$$
\begin{aligned}
L & =\left[\begin{array}{l}
E\left(z_{00}\right) \\
E\left(z_{11}\right) \\
E\left(z_{22}\right) \\
E\left(z_{33}\right)
\end{array}\right]=\left[\begin{array}{cccc}
E_{0}\left(z_{00}\right) & E_{1}\left(z_{00}\right) & E_{2}\left(z_{00}\right) & E_{0}\left(z_{00}\right) \\
E_{0}\left(z_{11}\right) & E_{1}\left(z_{11}\right) & E_{2}\left(z_{11}\right) & E_{0}\left(z_{11}\right) \\
E_{0}\left(z_{22}\right) & E_{1}\left(z_{22}\right) & E_{2}\left(z_{22}\right) & E_{0}\left(z_{22}\right) \\
E_{0}\left(z_{33}\right) & E_{1}\left(z_{33}\right) & E_{2}\left(z_{33}\right) & E_{0}\left(z_{33}\right)
\end{array}\right] \\
& =\left[\begin{array}{cccc}
1.0000 & -0.5000 & 0 & 0.2500 \\
1.0000 & -0.1667+0.3333 i & -0.3333-0.1111 i & 0.1759-0.2592 i \\
1.0000 & 0.1667+0.6667 i & -0.6666+0.2222 i & -0.3425-0.7407 i \\
1.0000 & 0.5000+1.0000 i & -1.0000+1.0000 i & -1.7500-1.0000 i
\end{array}\right]
\end{aligned}
$$

where

$$
E_{0}(z)=1, E_{1}(z)=z-1 / 2, E_{2}(z)=z^{2}-z, E_{3}(z)=z^{3}-(3 / 2) z^{2}+(1 / 4)
$$

According to (27), we obtain the coefficients matrix

$$
W=L\left(M^{T}\right)+P_{0} L=\left[\begin{array}{cccc}
0 & 1.0000 & -1.0000 & 0 \\
0.3333+0.3333 i & 0.8333+0.0555 i & -0.4074+0.5185 i & -0.8548-0.3610 i \\
0.6667+0.6667 i & 0.6666+0.5556 i & 0.6297+1.9260 i & -1.7346-0.0555 i \\
1.0000+1.0000 i & 0.5000+1.5000 i & -1.0000+2.0000 i & -3.7500+0.2500 i
\end{array}\right]
$$

For the initial condition $f(0)=-1$, we have 


$$
\begin{aligned}
{\left[U_{0} ; \alpha_{0}\right] } & =[E(0) ; S(0)] \\
& =\left[\begin{array}{llllll}
1.0000 & -0.5000 & 0 & 0.2500 & ; & -1
\end{array}\right] .
\end{aligned}
$$

Substituting the matrix form of initial conditions into $W$ and $S$, we obtain fundamental matrix

$$
\hat{W}=\left[\begin{array}{cccc}
0 & 1.0000 & -1.0000 & 0 \\
0.3333+0.3333 i & 0.8333+0.0555 i & -0.4074+0.5185 i & -0.8548-0.3610 i \\
0.6667+0.6667 i & 0.6666+0.5556 i & 0.6297+1.9260 i & -1.7346-0.0555 i \\
1.0000 & -0.5000 & 0 & 0.2500
\end{array}\right]
$$

and

$$
\hat{S}=\left[\begin{array}{c}
2.0000 \\
1.6667+0.1111 i \\
1.3333+1.1111 i \\
-1
\end{array}\right]
$$

Computing $F=(W)^{-1} \widehat{S}$, we have

$$
F=\left[\begin{array}{c}
-0.0000+0.0000 i \\
2.0000-0.0000 i \\
-0.0000-0.0000 i \\
-0.0000+0.0000 i
\end{array}\right]
$$

Substituting these coefficients into the Eq.(3), we obtain $f_{3}=E(z) F=2 z-1$ which is the exact solution of the given problem.

Example 2. Let us consider the following second order complex differential equation (5)

$$
f^{\prime \prime}(z)+z f^{\prime}(z)+z f(z)=e^{z}+2 z e^{z}
$$

under the initial conditions $f(0)=f^{\prime}(0)=1$.

Here $P_{0}(z)=P_{1}(z)=z$ and $S(z)=e^{z}+2 z e^{z}$.

We have collocation points

$$
z_{00}=0, z_{11}=(1+i) / 5, z_{22}=(2+2 i) / 5, z_{33}=(3+3 i) / 5, z_{44}=(4+4 i) / 5, z_{55}=1+i
$$

for $N=5$. Hence, we obtain

$$
P_{0}=P_{1}=\left[\begin{array}{llllll}
0 & 0 & 0 & 0 & 0 & 0 \\
0 & 0.2+0.2 i & 0 & 0 & 0 & 0 \\
0 & 0 & 0.4+0.4 i & 0 & 0 & 0 \\
0 & 0 & 0 & 0.6+0.6 i & 0 & 0 \\
0 & 0 & 0 & 0 & 0.8+0.8 i & 0 \\
0 & 0 & 0 & 0 & 0 & 1+i
\end{array}\right], \quad S=\left[\begin{array}{l}
1.0000 \\
1.5788+0.8185 i \\
2.0086+2.1449 i \\
2.0739+4.0681 i \\
1.4770+6.6318 i \\
-0.1686+9.7995 i
\end{array}\right] .
$$


Also, we can write matrix forms of initial conditions as follows:

$$
\begin{aligned}
& {[E(0) ; 1]=\left[\begin{array}{lllllll}
1.0000 & -0.5000 & 0 & 0.2500 & 0 & -0.5000 & ;
\end{array}\right]} \\
& {\left[E(0) M^{T} ; 1\right]=\left[\begin{array}{llllllll}
0 & 1.0000 & -1.0000 & 0 & 1.0000 & 0 & ; & 1
\end{array}\right]}
\end{aligned}
$$

We proceed as in the previous example by replacing rows, then we find the coefficients matrix of the approximate solution

$$
F=\left[\begin{array}{l}
1.7184-0.0000 i \\
1.7189+0.0003 i \\
0.8603+0.0021 i \\
0.2864+0.0056 i \\
0.0693+0.0048 i \\
-0.0107+0.0034 i
\end{array}\right] .
$$

The exact solution is given as $\mathrm{e}^{z}=\mathrm{e}^{x+i y}=e^{x} \cos (y)+i\left(e^{x} \sin (y)\right)$.

\begin{tabular}{|c|c|c|c|c|}
\hline $\mathrm{Z}$ & Exact solution & $\mathrm{N}=5$ & $\mathrm{~N}=6$ & $\mathrm{~N}=7$ \\
\hline $0.0+0.0 \mathrm{i}$ & $\begin{array}{l}0.000000000 \\
0.000000000 \mathrm{i}\end{array}$ & 0.0000000 & 0.0000000 & 0.0000000 \\
\hline $0.2+0.2 \mathrm{i}$ & $\begin{array}{l}0.242655268 \\
0.242655268 \mathrm{i} \\
\end{array}$ & $0.3947 \mathrm{E}-7$ & $0.1645 \mathrm{E}-7$ & $0.8650 \mathrm{E}-8$ \\
\hline $0.4+0.4 \mathrm{i}$ & $\begin{array}{l}0.580943900 \\
0.580943900 \mathrm{i} \\
\end{array}$ & $0.8493 \mathrm{E}-5$ & $0.2289 \mathrm{E}-6$ & $0.0254 \mathrm{E}-7$ \\
\hline $0.6+0.6 i$ & $\begin{array}{l}1.028845666 \\
1.028845666 \mathrm{i} \\
\end{array}$ & $0.1928 \mathrm{E}-4$ & $0.1325 \mathrm{E}-5$ & $0.3256 \mathrm{E}-7$ \\
\hline $0.8+0.8 \mathrm{i}$ & $\begin{array}{l}1.596505341 \\
1.596505341 \mathrm{i} \\
\end{array}$ & $0.0902 \mathrm{E}-3$ & $0.9232 \mathrm{E}-5$ & $0.0254 \mathrm{E}-5$ \\
\hline $1.0+1.0 \mathrm{i}$ & $\begin{array}{l}2.287355287 \\
2.287355287 \mathrm{i}\end{array}$ & $0.4977 \mathrm{E}-3$ & $0.5524 \mathrm{E}-4$ & $0.6659 \mathrm{E}-5$ \\
\hline
\end{tabular}

We compare this solution with the approximate solution for $N=5,6,7$ in Tables 1 .

Table 1. The Absolute Error for the Present Method at Various Points for Example 2

\section{Conclusions}

Complex differential equations are very complicated to obtain an analytic solution. So, researchers need to use some numerical methods to deal with them. We here offer Euler operational matrix method for solving high order complex differential equations with variable coefficients. Using collocation points with this method yields good results as in the given examples. Especially, if the considered problems have exact solution which is a polynomial of degree or less than ,then we can obtain the exact solution. In other cases, we can also have the approximate solutions which are compatible with the solution of the considered problem.

\section{References}

[1] Laine, I. (2008). Complex differential equations. Handbook of Differential Equations: Ordinary Differential Equations, 4, 269-363.

[2] Laine, I. (1993). Nevanlinna theory and complex differential equations. 15. Walter de Gruyter. 
[3] Hille, E. (1997). Ordinary differential equations in the complex domain. Courier Corporation.

[4] Heittokangas, J., Korhonen, R., \& Rattya, J. (2004). Growth estimates for solutions of linear complex differential equations. Annales-Academiae Sclentiarum Fennicae Mathematica, 29(1), Academia Scientiarum Fennica.

[5] Sezer, M., \& Mustafa, G. (2006). Approximate solution of complex differential equations for a rectangular domain with Taylor collocation method. Applied Mathematics and Computation, 177(2), 844-851.

[6] Toutounian, F., Emran, T., \& Stanford, S. (2013). A collocation method based on the Bernoulli operational matrix for solving high-order linear complex differential equations in a rectangular domain. Abstract and Applied Analysi. Hindawi Publishing Corporation.

[7] Bildik, N., \& Sinan, D. (2015). Implementation of Taylor collocation and Adomian decomposition method for systems of ordinary differential equations. Proceedings of the International Conference on Numerical Analysis and Applied Mathematics 2014 (ICNAAM-2014). AIP Publishing.

[8] Bildik, N., \& Deniz. S. (2015). Comparison of solutions of systems of delay differential equations using Taylor collocation method, Lambert W function and variational iteration method. Scientia Iranica. Transaction D, Computer Science \& Engineering, Electrical, 22(3), 1052.

[9] Deniz, S., \& Necdet, B. (2014). Comparison of Adomian decomposition method and Taylor matrix method in solving different kinds of partial differential equations. International Journal of Modeling and Optimization, 4(4), 292.

[10] Bildik, N., \& Sinan, D. (2013). Applications of Taylor collocation method and Lambert W function to systems of delay differential equations. TJMCS, 13.

[11] Srivastava, H. M., \& Pinter, A. (2004). Remarks on some relationships between the Bernoulli and Euler polynomials. Applied Mathematics Letters, 17(4), 375-380.

[12] Abramowitz, M., \& Irene, A. S. (1966). Handbook of mathematical functions. Applied Mathematics Series 55,62 .

[13] Srivastava, H. M. (2000). Some formulas for the Bernoulli and Euler polynomials at rational arguments. Mathematical Proceedings of the Cambridge Philosophical Society, 129(01), Cambridge University Press.

[14] Bildik, N., \& Sinan, D. (2015). On the asymptotic stability of some particular differential equations. International Journal of Applied Physics and Mathematics, 5(4), 252.

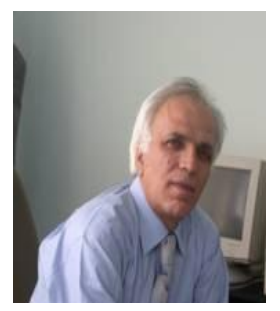

Necdet Bildik was born in Sivas, Turkey in 1951. He graduated from Ankara University in 1974. He earned the M.Sc. degree in University of Louisville, Kentucky, USA in 1978. He awarded the Ph.D. degree in Oklahoma State University, USA in 1982. He was assistant professor in 1988 and also he was became associate professor in 1995 . He was promoted to be professor in 2003. He is interested in numerical analysis, ordinary, partial and non-linear differential equations, ergodic theory, stability theory.

He has over than a hundred published articles in the national and international journals and conferences. He also serves as a reviewer for many international journals. 